\title{
How do interviewers and children discuss individual occurrences of alleged repeated abuse in forensic interviews?
}

\author{
Sonja P. Brubacher ${ }^{\mathrm{a}}$, Lindsay C. Malloy ${ }^{\mathrm{b}}$, Michael E. Lamb ${ }^{\mathrm{c}}$, and Kim P. Roberts ${ }^{\mathrm{d}}$ \\ ${ }^{\mathrm{a}}$ Central Michigan University, U.S.A. \\ ${ }^{\mathrm{b}}$ Florida International University, U.S.A. \\ ${ }^{\mathrm{c}}$ University of Cambridge, UK \\ ${ }^{\mathrm{d}}$ Wilfrid Laurier University, Canada
}

Manuscript submitted: August 20, 2012

Revision submitted: Dec 13, 2012

Word Count (excl. Tables, Figures, References): 5420

\section{Author Note.}

${ }^{\mathrm{c}}$ Correspondence and reprint requests should be addressed to Michael E. Lamb, Department of Psychology, University of Cambridge, Free School Lane, Cambridge CB2 3RQ, UK.

Mel37@cam.ac.uk. Preparation of this article was supported in part by a Social Sciences and Humanities Research Council Canada Graduate Scholarship-Michael Smith Foreign Study Supplement (SSHRCMSFSS) awarded to SPB as a PhD Candidate at Wilfrid Laurier University in 2010, and University Of Cambridge Vice-Chancellor's Discretionary Funds and a grant from the Nuffield Foundation to MEL. Portions of this research were presented to the Society for Applied Research in Memory and Cognition Conference (June 2011) and the $5^{\text {th }}$ Annual Meeting of the International Investigative Interviewing Research Group (May 2012). 


\begin{abstract}
Police interviews $(n=97)$ with 5- to 13-year-olds alleging multiple incidents of sexual abuse were examined to determine how interviewers elicited and children recounted specific instances of abuse. Coders assessed the labels for individual occurrences that arose in interviews, recording who generated them, how they were used, and other devices to aid particularisation such as the use of episodic and generic language. Interviewers used significantly more temporal labels than did children. With age, children were more likely to generate labels themselves, but most children generated at least one label. In $66 \%$ of the cases, interviewers ignored or replaced children's labels, and when they did so, children reported proportionately fewer episodic details. Children were highly responsive to the interviewers' language style. Results indicate that appropriately trained interviewers can help children of all ages to provide the specific details often necessary to ensure successful prosecution.
\end{abstract}

Keywords: interviewing, children, language style, repeated events, particularisation, child maltreatment 
Many victims of child sexual abuse are abused on multiple occasions (Connolly \& Read, 2006; Sas \& Cunningham, 1995). The most recent Canadian Incidence Study (CIS-4), for example, revealed that $51 \%$ of reported child sexual abuse cases involved multiple incidents (Trocmé et al., 2010). Because children are frequently the only witnesses to their abuse, their testimony is often crucial (McGough, 1994) and in many jurisdictions, they must 'particularise' specific incidents in some detail before offenders can be convicted (Guadagno, Powell, \& Wright, 2006; $R$ v. B. (G.), 1990; $S$ v. $R$, 1989). Although young victims may not be expected to provide exact dates and times for each occurrence, their accounts must include information about place, time, descriptions of participants, and/or other unique contextual details (Podirsky v R., 1990). How well do young victims provide this sort of particularising information, and what can investigative interviewers do to elicit episodic details (i.e., information specific to individual occurrences; Guadagno \& Powell, 2009, 2011; Guadagno et al., 2006)?

The aim of the current study was to investigate both interviewers' attempts to obtain, and children's provision of, episodic accounts of child sexual abuse in the course of formal forensic interviews. Specifically, our goals were to (1) describe both the types of labels (i.e., words used to delineate individual occurrences) generated and the ways in which these labels were used by both children and interviewers, and (2) characterize other techniques employed by interviewers to elicit episodic accounts, including prompting for episodic information and asking questions about event frequency and differences across occurrences.

\section{Particularization in Context}

Forensic interviews can be novel, confusing, and emotionally difficult experiences for the children involved (Poole \& Lamb, 1998; Wade \& Westcott, 1997). Best practice guidelines (e.g., Achieving Best Evidence, 2011) exhort investigative interviewers to obtain accounts in 
response to open-ended prompts which elicit information from recall rather than recognition memory. These types of prompts elicit more information per prompt (Hershkowitz, 2001; Lamb, Orbach, Hershkowitz, Esplin, \& Horowitz, 2007; Lamb, Sternberg, Orbach, Esplin, Stewart, \& Mitchell, 2003; Poole \& Lindsay, 1998), and also tend to be answered more accurately than option-posing (e.g., yes/no) and other closed-specific (e.g., "what colour was it?”) questions (Dent \& Stephenson, 1979; Goodman, Hirschman, Hepps, \& Rudy, 1991; Lamb \& Fauchier, 2001).

Even when open-ended questions elicit the desired narrative responses, however, the accounts may provide generic descriptions of 'what usually happened' when children have been abused on multiple occasions (e.g., Powell, Roberts, \& Guadagno, 2007). In order to recall an occurrence of repeated abuse, children must have the cognitive abilities to distinguish it from other occurrences, report details specific to that occurrence, and avoid confusing details across occurrences (see Roberts \& Powell, 2001 for a review). Accordingly, interviewers must be able to distinguish between generic accounts and reports of specific incidents, and to alter children's tendencies to report generic information by aiding them in isolating individual occurrences of abuse.

Isolating instances. Eliciting episodic details from children does not guarantee particularisation. Children typically have strong memories for content, and thus may be able to provide many episodic details without indicating how they relate to specific occurrences (Roberts \& Powell, 2001). Thus, interviewers must encourage children not only to retrieve episodic information but also to associate those details with specific incidents or events.

Powell and McMeeken (1998) recommended that, when children allege abuse on multiple occasions, unique labels should be associated with each incident remembered (e.g., "the 
first time"). In analogue research, the distinctive details typically relate to varying characteristics of the event scripts (e.g., children always wear a badge, but each day the type of badge changes). Children tend to remember these details well, but often confuse their association with other distinctive components of the events, for example reporting that a button badge was worn when they heard a story about a dog, whereas the button badge was worn on the second day and the story told on the third (Powell, Roberts, Ceci \& Hembrooke, 1999; Powell \& Thomson, 1996; Powell, Thomson \& Dietze, 1997). However, when Brubacher, Glisic, Roberts and Powell (2011) examined memories for completely unique details (those that were not part of the common event script), half of the 7- and 8-year olds reported them in their free narratives, and attributed them to the correct event nearly $75 \%$ of the time, although younger children (4- and 5year-olds) rarely reported such details. These data suggest that children as young as 7 years of age recognize that some occurrences of repeated events have distinctive features which can be used to label the individual events. In fact, children can be better than naïve interviewers at identifying unique labels for individual experienced events (Brubacher, Roberts, \& Powell, 2011 March).

Unfortunately, interviewers struggle to elicit effective labels from children in a nonsuggestive and non-leading manner (Powell et al., 2007). According to Powell et al. (2007), interviewers should ask clarifying questions when new details arise (e.g., "You said he took off your shirt. Is this still the time in the bathroom you're talking about, or another time?") and should consistently use the adopted labels, thereby reminding children to talk about specific instances. If children have difficulty retrieving episodic information, Yuille, Hunter, Joffe, and Zaparniuk (1993) recommended asking whether abuse ever happened in a different location or in a different manner to assist children in retrieving memories of incidents that may have unusual or 
unique features. However, interviewers questioning children about multiple incidents of abuse instead tend to ask questions suggesting the temporal source of content details, ask generic open questions, and ineffectively label specific events (Powell et al., 2007). No previous study has systematically characterized the labels arising in investigative interviews with respect to their types (e.g., temporal, location, suspect-related), by whom they were generated, how consistently they were used, and whether interviewers prompted children to describe incidents in distinctive ways. Accordingly, the present study was designed to address these issues by studying forensic interviews of young alleged abuse victims.

Using appropriate prompts. While generating labels for individual occurrences is helpful for particularization, interviewers must also prompt effectively. If multiple incidents are suspected, children should be asked about abuse frequency. Once it has been established that multiple incidents are alleged, recent research has demonstrated that interviewers should use episodic prompts when episodic responses are desired (Brubacher, Roberts, \& Powell, 2011; 2012; Schneider, Price, Roberts, \& Hedrick, 2011). Verbs in the past tense and some lexical markers (e.g., yesterday, the last time) characterize episodic information about individual occurrences, while use of the timeless present and the impersonal "you" pronoun indicate generic recall about what typically happened (Nelson \& Gruendel, 1981, 1986). Children tend to find it easier to talk generically about events for which they have well-established scripts or “general event representations" (Hudson, Fivush, \& Kuebli, 1992; Hudson \& Shapiro, 1991) and thus interviewers may need to refocus children on specific incidents by using episodic prompts. Although the recent research has shown that children tend to respond using the same level of language specificity as the interviewer (i.e., episodic or generic), it remains unknown how the focus of interviews shifts back and forth between levels of specificity and to what degree 
interviewers and children are responsible for these shifts. Accordingly, the current study provides the first descriptive account of these patterns of communication in forensic interviews.

\section{Current Study}

We sought to 1) describe how interviewers attempt to help children particularize incidents using labels, and describe other techniques interviewers use to help children particularize (e.g., by asking questions about event frequency and differences across occurrences), and 2) characterize the style of language (i.e., episodic or generic) used by children and interviewers so as to determine how each party influenced the style of language adopted. As this was the first examination of the labels used in forensic interviews and of the shifts between levels of specificity (episodic or generic), we did not make specific predictions with respect to these research questions. A secondary aim of the research was to confirm that children tend to respond to prompts using the same level of specificity as their interviewers (i.e., Brubacher et al., 2011, 2012; Schneider et al., 2011). It was thus expected that episodic prompts would be strongly associated with episodic child responses, while generic prompts would be associated with generic responses.

\section{Method}

\section{Sample}

The initial sample consisted of 105 forensic interviews of 5- to 13-year-old alleged victims of multiple incidents of child sexual abuse $(M$ age $=9.49, S D=2.45)$, conducted between 1997 and 2001 by 23 police officers in a UK police constabulary. All children were interviewed according to Memorandum of Good Practice Guidelines (Home Office/Department of Health, 1992, hereafter referred to as Memorandum) or the National Institute of Child Health and Human Development (NICHD) Investigative Interview Protocol (Lamb, Hershkowitz, 
Orbach, \& Esplin, 2008; Orbach, Hershkowitz, Lamb, Sternberg, Esplin, \& Horowitz, 2000) which is based upon similar scientific principles as the Memorandum but includes more precise guidelines. Differences associated with the type of interview conducted were not the focus of the current research, but were assessed in all analyses that follow and are reported when significant. Transcripts of eight interviews were excluded (two Memorandum): three because multiple incidents were not alleged, three because the children did not disclose abuse, and two because the children had language impairments. There were more boys in the excluded sample than expected by chance (four, or 50\% were boys), $\chi^{2}(1, N=105)=5.38, p=.02$, and the excluded sample was younger $(M=7.75, S D=1.83)$ than the final sample $(M=9.49, S D=$ $2.43), t(103)=1.98, p=.05$. Both children with language impairments were boys, as were two of the non-disclosing children. The final sample consisted of 81 girls and 16 boys; 37 were interviewed according to Memorandum guidelines and the remaining 60 in accordance with the NICHD Protocol.

\section{Coding}

All identifying information was removed from all interview transcripts prior to the research. The first two authors compiled a subset of transcripts for training/reliability assessment, selected randomly save to ensure that children of all ages were sampled.

Particularising occurrences. This coding focused on the techniques used by interviewers to help children describe individual occurrences of abuse. These included: adopting (or failing to adopt) children's labels for specific occurrences; and asking questions about abuse frequency and differences across occurrences.

Identifying incidents with labels. Coders noted each time the interviewer and/or child talked about a specific instance of abuse (rather than about what usually happened) by searching 
for episodic markers, including, but not limited to: specific information about time, place, perpetrator or child clothing, contextual information (e.g., "the time my mum was at the shops"), abusive acts, or any other details that distinguished any particular instance from the others. Coders then determined whether either the child or the interviewer, or both, had provided a specific label for the instance (e.g., "the last time") or a piece of unambiguously unique information that could be used as a label (e.g., "one time I pushed him so hard I fell [out of bed] onto the floor"). Specific labels were coded as: Temporal (e.g., "the day before Halloween," "it was on a Monday"); Locational (e.g., "the time at the building site"); Abuse-related (e.g. "the time he put his hand under my shirt"); and Situational (e.g., "the time my mum was at the shops," "the day I was home sick from school").

Coders also noted who initially provided the label. In order for a label to be judged as child-provided, the child had to use the detail in a labelling manner: "the day X" or "the time X." When the child provided episodic or unique details (such as the example concerning falling on to the floor) without specifically using the detail as a label, and the interviewer later prompted for more information about that time using the detail reported by the child, the label was coded as interviewer-provided.

Sometimes the labels for incidents were replaced by alternative labels, either by the original label-provider or by the other party. Changes in labels were recorded only when both labels unambiguously referred to the same instance. For example, one child reported "that time he put his hand under my shirt, and I just knew I had to tell someone." Later in the interview, it became apparent that the child reported the suspect after that incident, and so the "time he put his hand under my shirt" was also 'the last time', and the interviewer used the temporal label for the remainder of the interview. Although children typically accepted the label switch, they 
sometimes persisted in using their original labels. As such, all labels arising in the interview were retained and included in analyses, but they do not necessarily refer unambiguously to individual times. For example, a child alleging four instances of abuse could have more than four labels coded when either the interviewer or the child replaced one or more labels that had been used earlier.

Questions about the number of alleged incidents. Coders identified and tabulated interviewer prompts asking about the frequency of abuse or of specific abusive acts; responses to these questions were also used to estimate the number of allegations made by children. Other evidence for the number of allegations was gleaned from the remainder of the interview. For example, when a child responded to a frequency question by saying "a few times," then provided episodic information about three distinct occurrences, and said "no' when later asked whether it happened any other times, the child was coded as having alleged three occurrences of abuse. When the number of occurrences was not specified by the child or could not be estimated in this manner, frequency of allegations was coded as ambiguous.

Questions about differences among alleged incidents. Coders identified interviewer questions about differences among alleged incidents of abuse (most commonly, "Did anything different happen on that time [from other times]?"). Children's responses were coded as: 'difference reported', 'said it was always the same', 'don't know/don't remember', or digression.

Language specificity. To characterise interviewer language style in the allegation phase of the interview, each information-requesting interviewer prompt (see Hershkowitz, Orbach, Lamb, Sternberg, \& Horowitz, 2006) was coded as episodic, generic, or descriptive. Only the final prompt was coded when the interviewers asked more than one question in a conversational turn (see Lamb et al., 2003). Interrupted or unfinished interviewer prompts were not coded. 
Prompts were coded as episodic if they referred to specific events or occurrences (e.g., "What were you wearing?", "Tell me how he touched you on the last time"), as generic if they encouraged children to recall scripted/general information (e.g., "What did you usually wear?", "Tell me more about the touching [when not asked in the context of a specific time]"), and as descriptive when they requested semantic information such as the suspect's age and address, or an account of how the child and suspect came to be acquainted (see Brubacher et al., 2011; 2012; Schneider et al., 2011, for similar coding procedures).

Children's responses were first divided into units of information--phrases containing at least a subject and a verb (e.g., "I ran") and sometimes also objects, adjectives, and adverbs (e.g., "and then he slammed the bedroom door really loud"). Subjects/objects involved in the same behaviour were only counted as one unit (e.g., "Me, L, S and T were sitting on the floor" "He pulled off my trousers and pants"), but subjects/objects involved in a different behaviour were counted as an additional unit of information (e.g., "and G was sitting on the settee", "but he left my shirt on"). The units of information were then coded as episodic, generic, or descriptive using the same rules applied when coding interviewer prompts. For example, the child utterance “He does it always, like, when mum's at work, |but one day it happened when she went to the shop for ten minutes. | The shop's just across the street" contained three units, coded as generic, episodic and descriptive, respectively.

Conversational pattern analysis. We also coded the dyadic interaction between interviewer and child to elucidate patterns of mutual influence. Each time an individual switched to a different language coding category (i.e., episodic, generic or descriptive) and the other individual responded (e.g., child gave an episodic description then provided generic details, and interviewer responded with a follow-up prompt), we coded: (1) who initiated the switch - the 
child or the interviewer; 2) to what language style, and 3) with what language style the other party responded. There were thus 18 possible coding categories ( 3 possible initiating categories and 3 possible response categories for each of two initiating parties).

\section{Reliability}

Ten transcripts were randomly selected for training purposes (six Protocol, four Memorandum), and ten additional transcripts were used to estimate inter-coder reliability after training. The selected transcripts involved children of all ages. Three coders (including two of the authors) shared coding tasks about equally, with a fourth coder, who was otherwise not associated with the study, randomly selecting and assessing reliability on an additional ten transcripts after coding was complete. Reliability for all categorical variables was calculated using Kappa (overall range $=.72-1.00$ ), and reliability for codes representing quantities was calculated using percent agreement (overall range $=91-100$ ). All disagreements were resolved through discussion.

\section{Results}

\section{Particularising Occurrences}

Identifying incidents with labels. Five interviews contained no labels at all (three with 5-year-olds, one each with a 9- and 12-year-old; four Memorandum and one Protocol; all were coded as having alleged five or more instances of abuse). In the remaining interviews, 4.58 ( $S D$ $=2.68$ ) labels were generated on average; the numbers ranged from $1-13$ (although these did not necessarily characterize unique times; that is, original labels and replacements are included, and not all labelled occurrences were probed in detail).

Children spontaneously generated an average of $2.02(S D=1.944)$ labels per interview. A logistic regression analysis to explore factors associated with children's spontaneous provision 
of labels demonstrated that child age in years predicted the spontaneous provision of labels for incidents, $\chi^{2}(1)=7.20, p=.007$; it correctly classified $79.3 \%$ of the cases, Nagelkerk $r^{2}=.114$. None of the other participant variables (e.g., interview type, gender) were significant predictors, Wald's $\leq 1.97, p \mathrm{~s}=n s$. Figure 1 shows the proportion of labels generated by children (versus interviewers) for each age in years. The number of children at each age, and the number of interviews that contained at least one label, are reported below the figure.

Interviewers did not always adopt children's labels, however; in 27 (38\%) of the 71 interviews in which children provided at least one label, the interviewer replaced at least one of the child's labels, and in $11(15.5 \%)$ all of the children's labels were replaced or altered by interviewers. Sometimes interviewers chose to ignore details that might have been useful as labels for specific events: nearly half $(n=33,47 \%)$ of the 71 children who provided one or more labels had at least one label ignored. The greater the proportion of labels ignored, the less likely children were to follow the interviewers' language shifts, $r(69)=-.30, p=.012$.

Of all children in the sample, $47(66 \%)$ had at least one label either ignored or replaced. Surprisingly, child age in years was not correlated with the proportions of labels ignored or replaced, $r \mathrm{~s} \leq .144, p s=n s$ (see Figure 2), but the greater the proportion of children's labels that were ignored or replaced, the lower the proportion of episodic information provided, $r(69)=-$ $.261, p=.028$. Figure 2 also includes the raw number of labels produced by children at each age.

Figure 3 shows the types of labels introduced by interviewers and children, each expressed as a proportion of the total number of labels each produced. A paired-samples $t$-test revealed that proportionally more of the interviewers' labels $(M=.45, S D=.39)$ than of the children's labels $(M=.29, S D=.39)$ were temporal in nature, $t(61)=2.40, p=.019$, Cohen's d 
$=.41$, and there were no differences in the use of the less common Location-, Abuse- or Situation-related labels. It was not possible to compare interviewers' and children's labelling preferences in a single analysis because that would have required that both parties generate each type of label during the course of each interview.

Frequency of allegations. Most children (85\%) were asked at least one question about the frequency with which they had allegedly been abused. None of the participant variables (e.g., gender, interview type) were associated with the likelihood that they were asked, $\chi^{2} \mathrm{~s} \leq$ $3.59, p s=n s$, and age in years was not correlated with the number of frequency questions asked, $r(95)=.113, p=n s$.

The alleged frequencies ranged from two to multiple times per week over several years, with 31 cases in which the number of alleged incidents was ambiguous; these cases were thus excluded from the following four correlational analyses. The more incidents children alleged (from two, to five or greater, coded as 2,3,4, or 5), the greater the proportion of generic interviewer prompts (e.g., "what else does he do?"), $r(64)=.493, p<.001$. As they grew older, children were able to provide episodic (i.e., "particularising") details for more incidents, $r(64)=$ $.432, p<.001$, but there was no relation between age and the estimated number of incidents.

Questions about differences. The greater the number of incidents alleged, the more often interviewers asked about differences among times, $r(64)=.291, p=.018$, and the less likely they were to get episodic information from children, $r(64)=-.319, p=.009$. In addition, across the entire sample, the more interviewers asked whether there were differences among times, the less they used episodic prompts, $r(95)=-.285, p=.005$, and the more they used generic prompts, $r(95)=.273, p=.007$. 
Of the 19 children who were asked about differences, ten responded that it was always the same, while seven reported a difference, only three of which were unique to particular episodes (the others referred to "sometimes x, sometimes y"). One child did not answer the question, and another indicated that she could not remember. There were too few children per cell, however, to conduct additional statistical analyses.

\section{Language Specificity}

The levels of language specificity used by interviewers and children were strongly related, $r(95)=.943, p \mathrm{~s}<.001$; interviewers primarily used episodic prompts $(M=.68, S D=$ $.26)$, and children responded accordingly $(M=.70, S D=.26)$. The parties' use of generic language was similarly related, $r(95)=.950, p<.001$ (interviewer prompts $M=.25, S D=.25$; child utterances $M=.23, S D=.24$ ). Descriptive language was rare.

Protocol interviewers $(M=.74, S D=.22)$ used proportionally more episodic prompts than did Memorandum interviewers $(M=.58, S D=.29), t(61.55)=-2.93, p=.005$, Cohen's $d=$ .65 , and children's responses differed as well: child proportion episodic Protocol, $M=.76, S D=$ .20 ; Memorandum, $M=.59, S D=.30, t(55.15)=-3.06, p=.003$, Cohen's d $=.71$. Regardless of interview type, however, these features of interviewer and child language were strongly correlated, $r \mathrm{~s} \geq .897, p \mathrm{~s}<.001$.

Conversational pattern analysis. Child age was significantly correlated with the number of shifts coded, but inclusion of this variable as a covariate did not affect any of the results, so the results reported do not include this covariate. A 2 (Interview type: Memorandum v. Protocol) x 2 (Initiator: Child v. Interviewer) mixed model ANOVA revealed a significant effect of Initiator, $F(1,95)=274.14, p<.001, \eta_{\mathrm{p}}^{2}=.743$, and an Interview type $\mathrm{x}$ Initiator interaction, $F(1,95)=24.29, p<.001, \eta_{\mathrm{p}}{ }^{2}=.204$ on the number of shifts. To test the interaction, 
we conducted two independent-samples $t$-tests, one for each level of Initiator (alpha $=.025$ ).

Children initiated shifts as often in Protocol $(M=7.78, S D=5.50)$ and Memorandum $(M=7.11$, $S D=4.89)$ interviews, $t(95)<1, p=n s$; Cohen's $\mathrm{d}=.13$, but interviewers shifted significantly less often in Protocol $(M=15.43, S D=8.23)$ than in Memorandum interviews $(M=21.24, S D=$ 8.69), $t(95)=3.31, p=.001$, Cohen's $\mathrm{d}=.70$.

We next calculated the proportion of children's and interviewers' shifts that elicited congruent responses (e.g., child shift generic/interviewer responds generically) by dividing the number of congruent shifts by the total number of shifts for each party. A 2 (Interview Type) x 2 (Initiator) mixed model ANOVA revealed only a main effect for Initiator, $F(1,94)=165.50, p<$ $.001, \eta_{\mathrm{p}}{ }^{2}=.68$, indicating that children were significantly more likely to follow interviewer shifts $(M=.90, S D=.08)$ than interviewers were to follow children's shifts $(M=.51, S D=.27)$. Most shifts elicited congruent responses, and many types of shift-response patterns were quite rare (see Table 1). Table 1 shows that when children shifted to reporting generic information, interviewers followed up with a generic prompt $37 \%$ of the time, and redirected the children with an episodic prompt $51 \%$ of the time. As descriptive language was rarely used, shifts to this language style are omitted from Table 1.

\section{Discussion}

The current research was designed to describe 'particularization' as it happened naturally in the course of forensic interviews conducted in accordance with best-practice guidelines, and to identify effective strategies for interviewing children who have allegedly been sexually abused on multiple occasions. Our analyses showed that there were positive relations between the language styles used by children and interviewers supporting findings of previous analogue (Brubacher et al., 2011; 2012) and field (Schneider et al., 2011) studies. 
This research generated a number of novel findings. First, while children frequently responded to interviewer prompts using congruent language styles, interviewers often ignored the children's linguistic leads. Children were less likely to follow interviewer shifts, however, when interviewers ignored their attempts to provide labels, and the more often interviewers ignored children's labels or used different words to characterize occurrences, the lower the proportion of episodic details provided by children. Although children were increasingly likely to provide spontaneous labels with age, there was no age-related pattern in the extent to which interviewers ignored or replaced the children's labels. These novel data suggest new directions for laboratory analogue research and also have important implications for professionals who conduct interviews with children. It should be noted, however, that the sample of interviews examined here only included children who made allegations about sexual abuse, and these results may not generalize to situations in which other types of maltreatment are alleged (e.g., physical abuse, neglect).

\section{Isolating Incidents}

Particularisation requires that incidents must be contextualized in terms of time, place or some other unique contextual detail ( $S \vee R, 1989)$, and it can be challenging for interviewers to find 'labels' or names for each of many incidents (Powell et al., 2007). Ideally, children should label occurrences so that their memory searches are not biased by cues from interviewers, but interviewers may worry that children's labels are not sufficiently unique (Powell et al., 2007), and younger children may struggle to come up with labels on their own (Brubacher et al., 2011 March).

In the current field sample, $71(73 \%)$ of the children spontaneously provided at least one label for a specific incident of abuse. Yet, in $38 \%$ of those interviews, one or more labels were 
replaced by the interviewers. For example, a child who described an occurrence as the "time behind the shop", and later revealed that had been the first time, was prompted for the remainder of the interview to talk about the "first time." Interviewers tended to prefer temporal labels, using them significantly more often than did children. This is noteworthy because children's temporal understanding and use of time-related language develops gradually. Interviewers' use of temporal labels and their replacement of children's labels with temporal ones may present difficulties for young children in particular (Friedman, 2007; Friedman \& Lyon, 2005; Orbach \& Lamb, 2007). Sometimes interviewers ignored children's spontaneous labels, possibly thereby missing opportunities to probe other (perhaps better remembered) incidents. The more often interviewers ignored these attempts by children, the less often those children followed the interviewers' language shifts. Interviews that contained ignored or replaced labels were characterised by proportionally less episodic information being provided by the children. These findings further demonstrate the reciprocal influences that typify interview dynamics.

Recent research suggests other techniques with which interviewers might help children to label occurrences. For example, before adopting a child's label and using it to prompt for episodic detail about one occurrence of a lab-based repeated event, interviewers in Brubacher et al.'s (2011, March) study first asked children whether their label was a detail was relevant to any of the other occurrences. If so, the interviewer prompted for a different label. If the child could not provide a unique label spontaneously, the interviewer chose a detail previously mentioned by the child to serve as a label. The results were striking: 82 of 84 labels were unique, and the two that were not unique were chosen by the interviewers! Other aspects of this topic ripe for laboratory analogue research include examining the effects of replacing and/or ignoring children's labels on the amount and accuracy of information children report. 


\section{Using Appropriate Prompts}

All of the children in our sample alleged that they had been abused on multiple occasions, and $85 \%$ of them were asked specific questions about abuse frequency, in line with best-practice recommendations (Lamb et al., 2008), in comparison with only 55\% and 58\% in Guadagno and Powell's (2011) study of police officers observed in field and mock interviews, respectively.

As in lab-based research demonstrating that children readily report details that characterise most or all occurrences (e.g., Powell \& Roberts, 2002; Powell \& Thomson, 1996), the current study found that the greater the number of estimated experiences, the more likely interviews were to contain generic language. When interviews were dominated by generic prompts and responses, interviewers were more likely to ask about differences among occurrences, but the majority of these children responded that it was "always the same" (see also Guadagno \& Powell, 2011). Only 19 children were asked about differences so further analyses were not possible, but it would be valuable to conduct controlled lab research on the utility of questioning children about differences among individual event occurrences.

Although interviewers did prompt children generically, their tendency was to ask for episodic information, and to refocus children who provided generic details by following up their utterances with an episodic prompt. In the current study, we provide the first published analysis of interviewers and children shifting from one language style to another (e.g., child speaking generically about "what usually happens" and interviewer following up with a prompt for specific episodic information) in order to determine which party was 'driving' the interview language style. Interviewers were in the driver's seat; they switched the language specificity of the interviews significantly more often than did children, and, while they only followed children's shifts $51 \%$ of the time, children shifted accordingly in response $90 \%$ of the time. 
Results suggest that when children are alleging multiple incidents of abuse, interviewers must balance the desire to allow children to report as freely as possible with the need to elicit specific episodic information.

\section{Conclusions and Future Directions}

The present study provides a unique characterization of how interviewers and children attempted to particularise individual incidents of sexual abuse in forensic interviews by describing the types of labels generated and how they were used during the course of the investigative interviews, as well as the reciprocal influences of interviewers' prompts and children's responses on the specificity of the children's reports. Findings are of interest to those who study the organization of children's memory and event narratives and to those who must question children in forensic contexts. The present study suggests several new lines of inquiry for both field and lab-based research exploring the potential cognitive and motivational effects of ignoring and/or replacing children's labels; asking whether doing so decreases children's accuracy or willingness to provide information, and determining whether questioning about differences among incidents produces new episodic leads. Empirical study of these issues has the potential to answer further questions about memory organization for repeated events, and inform and improve multiple-incident interviewing of child victims/witnesses. 


\section{References}

Brubacher, S.P., Glisic, U., Roberts, K.P., \& Powell, M.B. (2011). Children's ability to recall unique aspects of one occurrence of a repeated event. Applied Cognitive Psychology, 25, 351-358. doi: 10.1002/acp.1696

Brubacher, S.P., Roberts, K.P., \& Powell, M.B. (2011). Effects of practicing episodic versus scripted recall on children's subsequent narratives of a repeated event. Psychology, Public Policy, and Law, 17, 286 - 314. doi: 10.1037/a0022793

Brubacher, S.P, Roberts, K. P., \& Powell, M.B. (2011, March). Training children with repeatedevent experience to label unique aspects of occurrences. Paper presented in K. London (Discussant). Motivational and cognitive influences on children's testimony. Symposium at the annual meeting of the American Psychology-Law Society/4th International Congress on Psychology and Law, Miami, FL.

Brubacher, S.P., Roberts, K.P., \& Powell, M.B. (2012). Retrieval of episodic versus generic information: Does the order of recall affect the amount and accuracy of details reported by children about repeated events? Developmental Psychology, 48, 111-122. doi:

\section{$10.1037 / \mathrm{a} 0025864$}

Connolly, D.A., \& Read, J.D. (2006). Delayed prosecutions of historic child sexual abuse: Analyses of 2064 Canadian criminal complaints. Law and Human Behavior, 30, $409-$ 434. doi: 10.1007/s10979-006-9011-6

Dent, H. R., \& Stephenson, G. M. (1979). An experimental study of the effectiveness of different techniques of questioning child witnesses. British Journal of Social \& Clinical Psychology, 18, 41-51. doi:10.1111/j.2044-8260.1979.tb00302.x 
Friedman, W. J. (2007). The development of temporal metamemory. Child Development, 78, 1472-1491. doi:10.1111/j.1467-8624.2007.01078.x

Friedman, W. J., \& Lyon, T. D. (2005). Development of temporal-reconstructive abilities. Child Development, 76, 1202-1216. Retrieved from http://search.proquest.com/docview/620978650?accountid=15090

Goodman, G. S., Hirschman, J. E., Hepps, D., \& Rudy, L. (1991). Children's memory for stressful events. Merrill-Palmer Quarterly, 37, 109-157.

Guadagno, B.L., \& Powell, M.B. (2009). A qualitative examination of police officers’ questioning of children about repeated events. Police Practice and Research: An International Journal, 10, 61 - 73. doi: 10.1080/15614260802128468

Guadagno, B. L., \& Powell, M. B. (2011). Child abuse and sex crimes: Examining police officer interview techniques involving juvenile victims. In J. F. Albrecht \& D. Das (Eds.), Effective Crime Reduction Strategies: International Perspectives (pp. 551-567). Boca Raton, US: CRC Press.

Guadagno, B., Powell, M. B., \& Wright, R. (2006). Police officers' and legal professionals' perceptions regarding how children are, and should be, questioned about repeated abuse. Psychiatry, Psychology \& Law, 13, 251-260. doi: 10.1375/pplt.13.2.251

Hershkowitz, I. (2001). Children's responses to open-ended utterances in investigative interviews. Legal and Criminological Psychology, 6, 49-63. doi:

$$
10.1348 / 135532501168190
$$

Hershkowitz, I., Orbach, Y., Lamb, M. E., Sternberg, K. J., \& Horowitz, D. (2006). Dynamics of forensic interviews with suspected abuse victims who do not disclose abuse. Child Abuse \& Neglect, 30, 753-769. doi:10.1016/j.chiabu.2005.10.016 
Home Office/Department of Health. (1992) Memorandum of Good Practice on Video Recorded Interviews with Child Witnesses for Criminal Proceedings. London: HMSO.

Hudson, J.A., Fivush, R., \& Kuebli, J. (1992). Scripts and episodes: The development of event memory. Applied Cognitive Psychology. Special Issue: Memory in everyday settings, 6, 483-505. doi: 10.1002/acp.2350060604

Hudson, J.A., \& Shapiro, L.R. (1991). From knowing to telling: The development of children's scripts, stories, and personal narratives. In A. McCabe \& C. Peterson (Eds.), Developing Narrative Structure (pp. 89 - 136). Hillsdale, NJ: Erlbaum.

Lamb, M. E. \& Fauchier, A. (2001), The effects of question type on self-contradictions by children in the course of forensic interviews. Applied Cognitive Psychology, 15, 483-491. doi: 10.1002/acp.726

Lamb, M. E., Hershkowitz, I., Orbach, Y., \& Esplin, P. W. (2008). Tell me what happened: Structured investigative interviews of child victims and witnesses. Chichester UK: Wiley.

Lamb, M.E., Orbach, Y., Hershkowitz, I., Esplin, P.W., \& Horowitz, D. (2007). A structured forensic interview protocol improves the quality and informativeness of investigative interviews with children: A review of research using the NICHD protocol. Child Abuse \& Neglect, 31, 1201-1231. doi: 10.1016/j.chiabu.2007.03.021

Lamb, M.E., Sternberg, K.J., Orbach, Y., Esplin, P.W., Stewart, H., \& Mitchell, S. (2003). Age differences in young children's responses to open-ended invitations in the course of forensic interviews. Journal of Consulting and Clinical Psychology, 71, 926 - 934. doi: 10.1037/0022-006X.71.5.926

McGough, L.S. (1994). Child witnesses: Fragile voices in the American legal system. New Haven, CT, US: Yale University Press. 
Nelson, K., \& Gruendel, J. (1981). Generalized event representations: Basic building blocks of cognitive development. In M.E. Lamb \& A.L. Brown (Eds.), Advances in Developmental Psychology, Vol. 1 (pp. 131 - 158). Hillsdale, NJ: Erlbaum.

Nelson, K. \& Gruendel, J. (1986). Children's scripts. In J. Gruendel (Ed.), Event knowledge: Structure and Function in Development (pp. 21-46). Hillsdale, NJ: Erlbaum.

Office for Criminal Justice Reform (2011). Achieving Best Evidence in Criminal proceedings: Guidance for vulnerable or intimidated witnesses, including children. London, UK: Office for Criminal Justice Reform.

Orbach, Y., Hershkowitz, I., Lamb, M. E., Sternberg, K. J., Esplin, P. W., \& Horowitz, D. (2000). Assessing the value of structured protocols for forensic interviews of alleged abuse victims. Child Abuse and Neglect, 24, 733-752. doi: 10.1016/S01452134(00)00137-X

Orbach, Y. \& Lamb, M. E. (2007), Young children's references to temporal attributes of allegedly experienced events in the course of forensic interviews. Child Development, 78, 1100-1120. doi: 10.1111/j.1467-8624.2007.01055.x

Podirsky v. R. (1990). 3 WAR 128.

Poole, D.A., \& Lamb, M.E. (1998). Investigative interviews of children: A guide for helping professionals. Washington DC, US: APA, 295 pages.

Poole, D. A., \& Lindsay, D.S. (1998). Assessing the accuracy of young children's reports: Lessons from the investigation of child sexual abuse. Applied and Preventive Psychology, 7, 1-26. doi:10.1016/S0962-1849(98)80019-X

Powell, M.B. \& McMeeken, L. (1998). “Tell me about the time when...”: 9 Golden Rules for interviewing a child about a multiple offence. Australian Police Journal, 52, 104 - 108. 
Powell, M. B. \& Roberts, K. P. (2002), The effect of repeated experience on children's suggestibility across two question types. Applied Cognitive Psychology, 16, 367-386. doi: 10.1002/acp.801

Powell, M.B., Roberts, K.P., Ceci, S.J., \& Hembrooke, H. (1999). The effects of repeated experience on children's suggestibility. Developmental Psychology, 35, 1462-1477. doi: $10.1037 / 0012-1649.35 .6 .1462$

Powell, M. B., Roberts, K. P., \& Guadagno, B. (2007). Particularisation of child abuse offences: Common problems when questioning child witnesses. Current Issues in Criminal Justice, 19, 64-74.

Powell, M.B., \& Thomson, D.M. (1996). Children's memory of an occurrence of a repeated event: Effects of age, repetition, and retention interval across three question types. Child Development, 67, 1988-2004. doi: 10.2307/1131605

Powell,M. B., Thomson, D. M. \& Dietze, P. M. (1997). Children's ability to remember an occurrence of a repeated event. Expert Evidence, 5, 133-139.

R. v. B. (G.) (1990). 2 S. C. R. 30.

Roberts, K. P., \& Powell, M. B. (2001). Describing individual incidents of sexual abuse: A review of research on the effects of multiple sources of information on children's reports. Child Abuse \& Neglect, 25, 1643-1659. doi: 10.1016/S0145-2134(01)00290-3

$S$ v. $R(1989)-168 C L R 266$

Sas, L. D., \& Cunningham, A. H. (1995). Tipping the balance to tell the secret: The public discovery of child sexual abuse. London, Ontario: London Family Court Clinic. 
Schneider, L., Price, H.L., Roberts, K.P., \& Hedrick, A. (2011). Children’s episodic and generic reports of alleged abuse. Applied Cognitive Psychology, 25, 862 - 870. doi: 10.1002/acp. 1759

Trocmé, N., Fallon, B., MacLaurin, B., Sinha, V., Black, T. Fast, E., ...\& Holroyd, J. (2010). Canadian Incidence Study of reported child abuse and neglect 2008 (CIS-2008): Major findings. Public Health Agency of Canada, Ottawa ON: 122 pages. doi: 978-1-10016915-6

Wade, A. \& Westcott, H.L . (1997) ‘No easy answers: Children’s perspectives on investigative interviews.' In H.L. Westcott and J. Jones (Eds.), Perspectives on the Memorandum. Policy, Practice and Research in Investigative Interviewing. Aldershot, UK: Arena.

Yuille, J.C., Hunter, R., Joffe, R., \& Zaparniuk, J. (1993). Interviewing children in sexual abuse cases. In Goodman G. S., Bottoms B. L. (Eds.), Child victims, child witnesses: Understanding and improving children's testimony (pp. 95-115). New York, US: Guilford Press. 


\section{Table 1}

Initiator-response patterns for episodic and generic language shifts.

\begin{tabular}{|c|c|c|c|c|c|c|}
\hline Initiator & Shift to & Response & $M$ & $S D$ & Range & $N$ \\
\hline \multirow[t]{6}{*}{ Child } & Episodic & Episodic & .71 & .33 & $0-1.00$ & 73 \\
\hline & & Generic & .14 & .25 & $0-1.00$ & 73 \\
\hline & & Descriptive & .04 & .15 & $0-1.00$ & 73 \\
\hline & Generic & Episodic & .51 & .40 & $0-1.00$ & 70 \\
\hline & & Generic & .37 & .36 & $0-1.00$ & 70 \\
\hline & & Descriptive & .05 & .12 & $0-.05$ & 70 \\
\hline \multirow[t]{6}{*}{ Interviewer } & Episodic & Episodic & .89 & .13 & $.50-1.00$ & 95 \\
\hline & & Generic & .06 & .10 & $0-1.00$ & 95 \\
\hline & & Descriptive & .01 & .04 & $0-1.00$ & 95 \\
\hline & Generic & Episodic & .09 & .18 & $0-1.00$ & 87 \\
\hline & & Generic & .87 & .24 & $0-1.00$ & 87 \\
\hline & & Descriptive & .01 & .06 & $0-.50$ & 87 \\
\hline
\end{tabular}

Note: Response categories for each shift do not total 1.00 because, in rare cases, interviewers prompted for, or children responded with, information unrelated to the abuse allegations (i.e., digressions). 


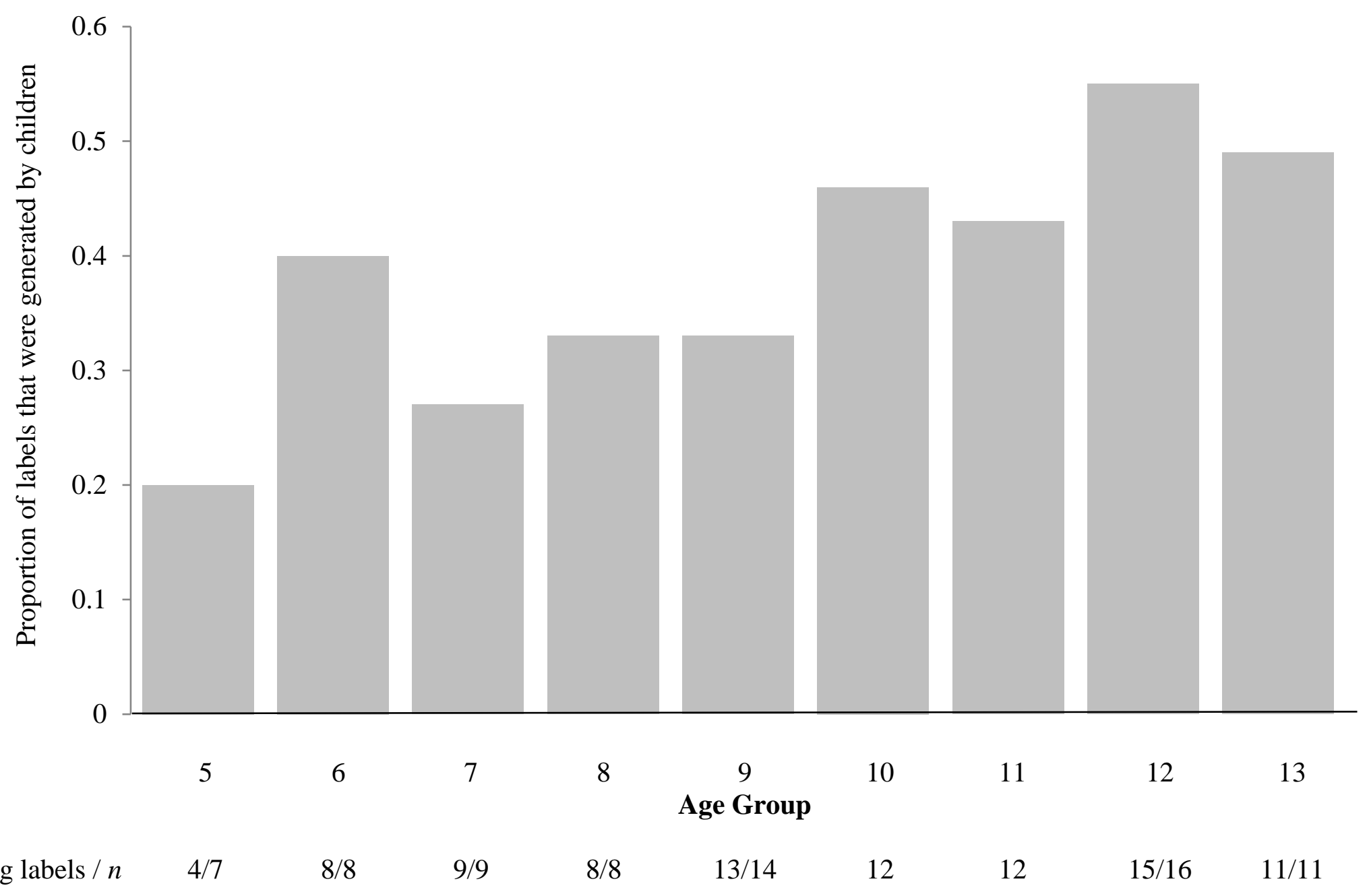

Figure 1 Proportion of labels in interviews that were generated by children. 


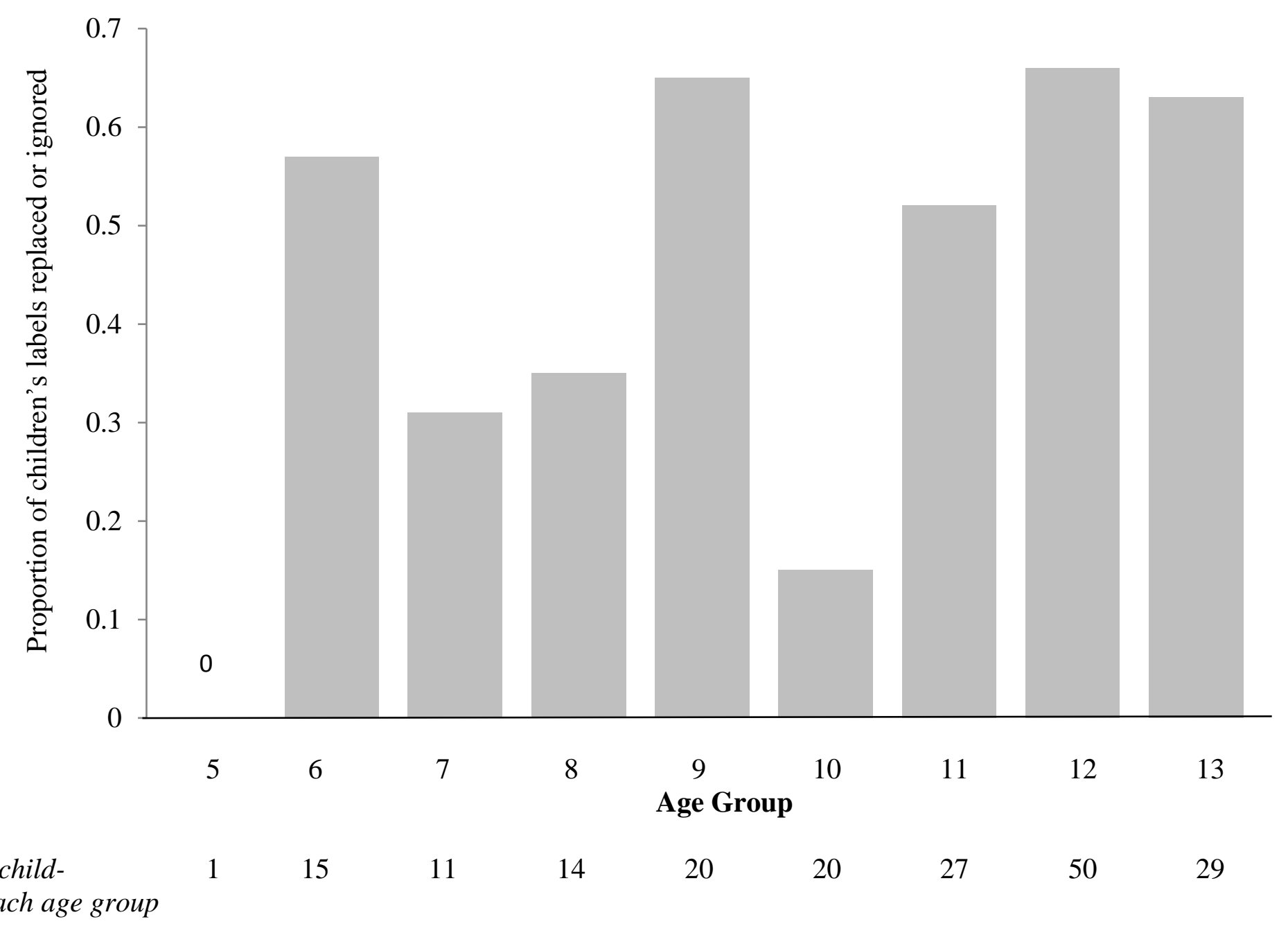

provided labels for each age group

Figure 2 Proportion of children's labels that were ignored or replaced by interviewers. 


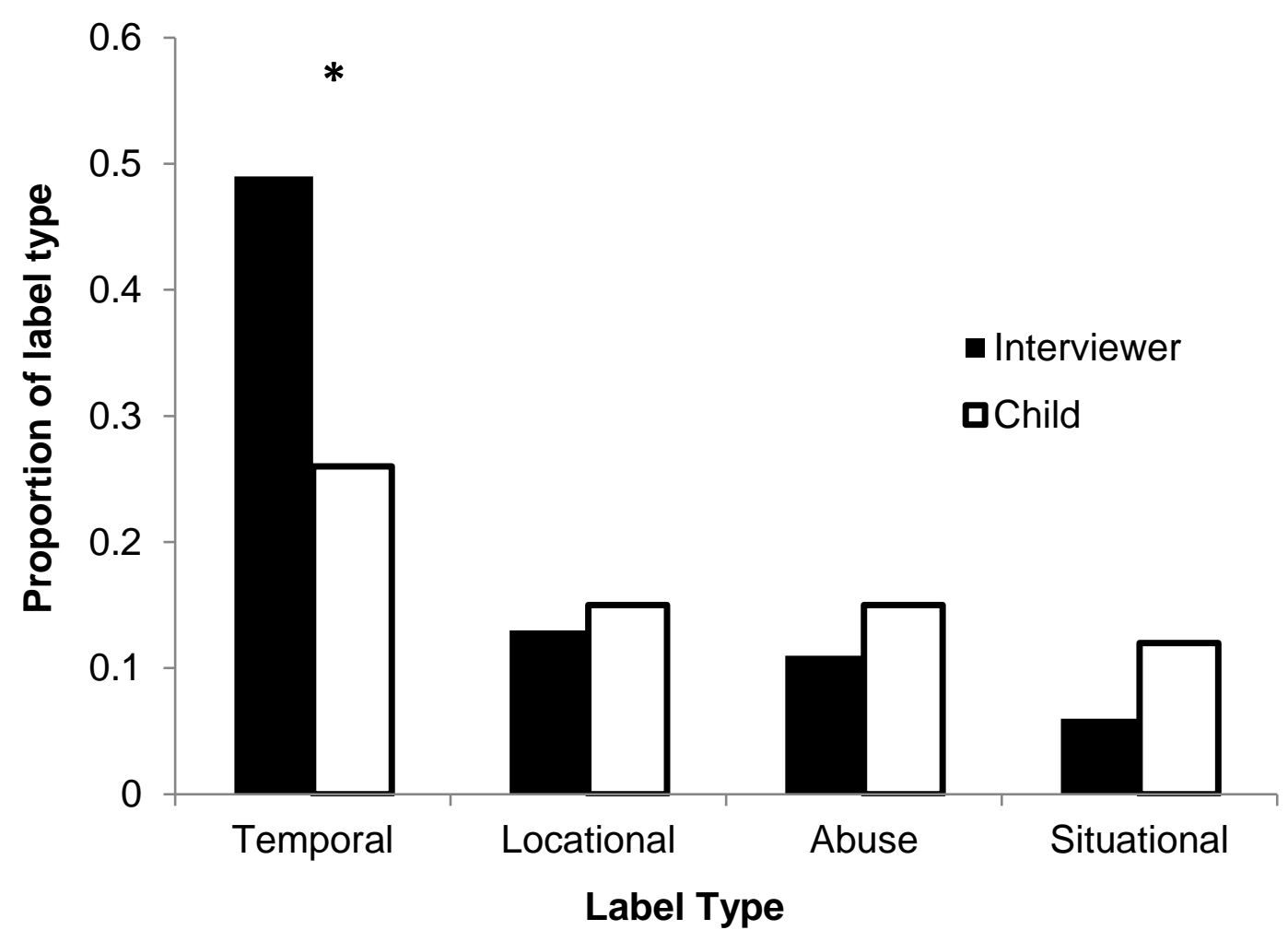

Figure 3 Types of labels produced by interviewers and children. 